\title{
Estratégias tecnológicas para formulações de anfotericina $B$ em sistemas lipídicos disponíveis no mercado farmacêutico e outros promissores sistemas de administração
}

\author{
Technological strategies for amphotericin B formulations \\ in lipid systems available in the pharmaceutical \\ market and other promising delivery systems
}

Recebido em: 02/10/2015 Aceito em: $30 / 05 / 2016$
Gustavo Julio Borges ARRUDA ${ }^{1}$; Gustavo ROSSANEZI ${ }^{1}$; Maria Virginia SCARPA $^{1}$; Erivaldo Socrates Tabosa do EGITO ${ }^{2}$; Anselmo Gomes de OLIVEIRA ${ }^{1}$ ${ }^{1}$ Faculdade de Ciências Farmacêticas de Araraquara, Universidade Estadual Paulista "Júlio de Mesquita Filho"- FCFar/UNESP. Rodovia Araraquara-Jaú, km 1, Campus Ville, Araraquara, SP. CEP-14801-902, Brasil,; ${ }^{2}$ Departamento de Farmácia, Centro de Ciências da Saúde, Universidade Federal do Rio Grande do Norte Rua Gal. Gustavo Cordeiro de Farias, S/N, Petrópolis Natal, RN. CEP 59010-180, Brasil;. E-mail: grossanezi@hotmail.com

\section{ABSTRACT}

Amphotericin $\mathrm{B}(\mathrm{AmB})$ is an antifungal reference drug in treatment of systemic mycoses since its discovery in the years 1950. The aim of this study was to establish the state of the art of the technological strategies involved in the administration drug dosage forms $\mathrm{AmB}$ and the the paths for intervention to improve the drug solubility, allowing its administration intravenously and minimize its toxicity. The importance of the studies to the medical-pharmaceutical field is related to new drug delivery systems that can increase membership by children and the elderly, in addition to increasing the therapeutic arsenal available to the medical clinic. Due the limited clinical utility of the normal AmB formulations because of its high toxicity, the new administration systems of AmB able to direct the antibiotic directly to site of infections has been the most studied. Therefore, the need for different vehicles other than the deoxycholate (Fungizon $\left({ }^{\circledR}\right.$ ) has been the most common alternative. The lipid formulations have been shown to be more effective and less toxic when compared to $\mathrm{AmB}$ deoxycholate, but not yet introduced to hospital routine for treating deep mycoses.

Keywords: amphotericin B; drug delivery systems; solubility; toxicity; systemic antifungal

\section{RESUMO}

A anfotericina $\mathrm{B}(\mathrm{AmB})$ é um fármaco antifúngico utilizado no tratamento de micoses sistêmicas desde sua descoberta nos anos de 1950. O objetivo deste trabalho foi estabelecer o estado da arte das estratégias tecnológicas envolvidas nas formas de administração da $\mathrm{AmB}$, nas intervenções diretas nos aspectos de melhoria de solubilidade do fármaco, que possibilitem sua administração por via intravenosa (iv) e minimizem sua toxicidade. Devido à limitada utilidade clínica do sal de desoxicolato (Fungizon ${ }^{\circledR}$ ) em razão de sua alta toxicidade, sistemas de liberação mais eficientes foram desenvolvidos. Neste trabalho, são apresentadas as principais formulações disponíveis no mercado farmacêutico e outras formulações lipídicas promissoras, as quais se mostraram mais eficazes como veículos de solubilização e menos tóxicas quando comparadas com a AmB desoxicolato, mas ainda não fazem parte da rotina hospitalar para $o$ tratamento de micoses profundas.

Palavras-chave: anfotericina B; sistemas de liberação; solubilidade; toxicidade; antifúngico sistêmico 


\section{INTRODUÇÃO}

O antibiótico antifúngico foi descoberto em 1955,no Instituto Squibb para Pesquisas Médicas, a partir de amostras de solo do Rio Orinoco da Venezuela. Em 1956, a anfotericina B (AmB) foi isolada numa forma mais pura, a qual continha apenas de 1 a $2 \%$ de anfotericina A. Somente em 1958 foi utilizada pela primeira vez no tratamento da paracoccidioidomicose e, posteriormente, em tratamentos de infecções causadas por outras espécies de fungos (1, 2). Seu espectro antifúngico e eficácia a colocaram na posição de fármaco de referência no controle de infecções fúngicas sistêmicas (Figura 1). Somente no ano de 1959 foi obtida a patente da AmB, sendo considerada um dos antifúngicos mais eficientes para o tratamento de micoses sistêmicas $(3,4,5,6)$. No final dos anos 50 a AmB já era utilizada na clínica médica, tendo sido o primeiro antifúngico a ser aprovado pela United US Food and Drug Administration (FDA) em 1965 (7, 8).

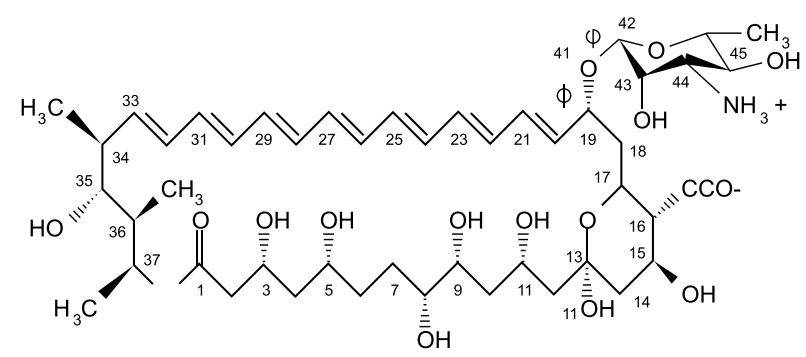

Figura 1 - Estrutura química da anfotericina B em meio neutro. Adaptado de Baginski et al., 1997 (26)

$\mathrm{O}$ mecanismo de ação da $\mathrm{AmB}$ está relacionado com uma interação específica com o ergosterol, esteroide constituinte da parede celular fúngica, ocorrendo a formação de poros através de membranas lipídicas que formam canais transmembrânicos $(9$, 10). A célula despolariza-se, tendo a sua permeabilidade alterada por meio do escape de pequenos íons e metabólitos (cátions monovalentes, prótons), principalmente íons potássio (Figura 2). Ligações intermoleculares de hidrogênio com grupos carboxila, amina primaria e hidroxila, estabilizam o canal na forma aberta, e assim, o conteúdo plasmático pode ser liberado, levando à perda da atividade celular e eventualmente à morte celular $(10,11)$.

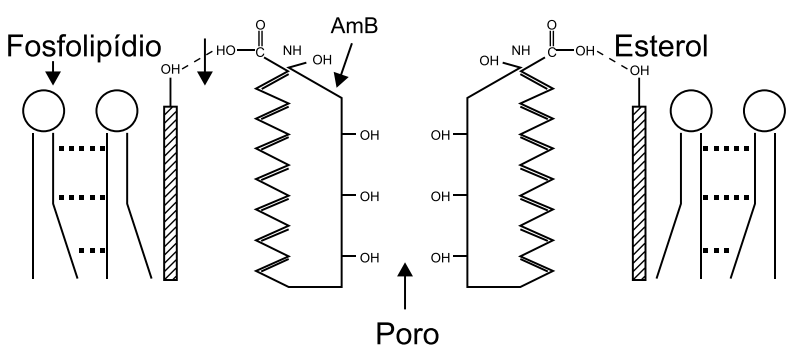

Figura 2 - Representação esquemática da interação da $A m B$ com o ergosterol na bicamada lipídica da membrana celular do fungo. Adaptado de Golenser \& Domb, 2006 (18).

Embora a AmB possua maior afinidade pelo ergosterol, muitos dos efeitos tóxicos que lhe são atribuídos são em decorrência da ligação ao colesterol e outros constituintes da membrana celular de mamíferos $(12,13)$.

A AmB é pouco solúvel na maioria dos solventes orgânicos, assim como na água. É solúvel em solventes apróticos, tais como o dimetilsulfóxido e dimetilformamida. Além da baixa solubilidade em água, também é muito pouco absorvida por via oral sendo, por isso, administrada rotineiramente por via i.v (14). Sua massa molecular é da ordem de $924,1 \mathrm{~g} / \mathrm{mol}$. A atividade antifúngica é máxima na faixa de $\mathrm{pH}$ entre 6,0 e 7,5, podendo ter ação fungistática ou fungicida, dependendo da concentração plasmática e tecidual do antifúngico e da susceptibilidade do patógeno (15).

Quando em concentração acima da concentração micelar crítica (CMC), as moléculas da AmB se auto associam formando dímeros ou agregados maiores (16). Tanto a forma monomérica quanto a agregada, podem formar canais em membranas celulares contendo ergosterol. No entanto, somente a forma agregada tem a capacidade de formar tais canais em membranas contendo colesterol, fenômeno responsável pela toxicidade, em células de mamíferos (17). Assim, uma das estratégias eficientes para diminuir a toxicidade da $\mathrm{AmB}$ em células de mamíferos seria o desenvolvimento de sistemas de administração capazes de imobilizar a AmB monomérica em suas estruturas, tornando-a menos tóxica para as membranas de células de mamíferos (que contém colesterol) e mais tóxicas para as células fúngicas, as quais contém ergosterol (15).

O uso clínico da $\mathrm{AmB}$, muitas vezes tem sido limitado pelos efeitos tóxicos e pela necessidade de aplicação i.v. A associação da AmB com sistema micelar de desoxicolato de sódio (DOC) por exemplo, proporciona um sistema estável do ponto de vista químico e físico-químico (AmB-DOC), possível de administração i.v., mas que possibilita pouca redução da toxicidade in 
vivo. Uma fração da AmB, dissolvida nas micelas, geralmente é complexada por liproteinas e metabolizada preferencialmente no fígado, aumentando o potencial de toxicidade do antibiótico (16). Com essa arquitetura, a primeira formulação comercial da AmB-DOC em meio tamponado surgiu em 1958, denominada de Fungizone ${ }^{\circledR}$ ou Fungizon ${ }^{\circledR}$. Trata-se de uma formulação contendo o tensoativo DOC em solução aquosa de glicose, formando uma dispersão micelar coloidal. Porém, devido ao seu potencial de toxicidade, a dose máxima recomendada de $1,5 \mathrm{mg} / \mathrm{kg} / \mathrm{dia}$, as quais algumas vezes não é suficiente para o tratamento completo (18).

Atualmente, outro sistema organizado, com fundamento semelhante ao das micelas, mas com maior potencial de solubilização da $\mathrm{AmB}$ são as microemulsões, as quais possuem um volume local bem superior aos dos sistemas micelares, disponível para a solubilização. Os fármacos ficam compartimentalizados nas gotículas da fase interna oleosa, a qual por apresentar propriedades físico-químicas bastante diferentes das do meio dispersante, promovem modificações nas propriedades biofarmacêuticas $(19,20)$. Formam-se sistemas reservatórios que modificam a velocidade de liberação de fármacos. Estes sistemas podem alterar os parâmetros farmacocinéticos, aumentar o índice terapêutico, e como consequência, diminuir a toxicidade e aumentar a eficácia clínica da AmB $(21,22,23,24)$. Entre os sistemas de liberação comerciais para a AmB, destacam-se o Ambisome $\AA$, formulação lipossomal que permite administrar dose de até 7,5 $\mathrm{mg} / \mathrm{kg} /$ dia com um perfil de segurança adequado, maior do que a fórmula convencional de Fungizone ${ }^{\circledR}(18)$.

A alta toxicidade da AmB de formulações convencionais e a baixa solubilidade em água determinam um perfil de absorção insignificante por via oral. Essas limitações tem estimulado a pesquisa de sistemas com habilidade de diminuir a toxicidade, como por exemplo, as formulações lipídicas (4). Entre as opções de formulações lipídicas para a veiculação da $\mathrm{AmB}$, existem as emulsões lipídicas, complexos lipídicos, soluções micelares, microemulsões e nanopartículas (25).

\section{TECNOLOGIAS COMPETITIVAS}

Aumento da solubilidade aparente da AmB. As formulações convencionais comercialmente utilizadas, denominadas de Fungizon ${ }^{\circledR}$ e Anforicin ${ }^{\circledR}$, são constituídas de $50 \mathrm{mg}$ de $\mathrm{AmB}$, aproximadamente 41 mg de DOC e 20,2 mg de tampão fosfato. O DOC é empregado para a solubilização micelar da AmB. O sistema não é homogêneo, podendo apresentar em sua constitui- ção três diferentes formas moleculares da $\mathrm{AmB}$, como a monomérica, oligomérica e agregados de AmB-DOC misturados com micelas vazias de DOC (17). Este medicamento apresenta-se na forma sólida como pó liofilizado para reconstituição e administração por via intravenosa. A eficácia clínica do medicamento é limitada pela toxicidade e efeitos colaterais já conhecidos, os quais se desenvolvem após algumas semanas de tratamento, além de problemas como baixa adesão dos pacientes ao tratamento devido à necessidade de hospitalização e dos altos custos $(27,28,29)$ Estudos revelam que a terapia medicamentosa dessas patologias oportunistas tratadas distantes do ambiente hospitalar oferece muitas vantagens psicológicas e econômicas ao paciente (30).

A baixa solubilidade em água e em muitos solventes aquosos constitui um sério problema para a definição de uma forma de administração da AmB. O antifúngico possui tendência de se autoassociar formando dímeros ou trimeros, com maior dificuldade solubilização em água e tampões aquosos (31).

Dessa forma, a associação da AmB com compostos tensoativos, tais como, laurilsulfato de sódio, sais de ácidos biliares como o desoxicolato de sódio, derivados de ciclodextrinas, colesterol e outros agregados supramoleculares constituem algumas das alternativas capazes de proporcionar diferentes formas de administração da $\operatorname{AmB}(15,32)$.

Estudos mostraram que através da utilização do laurato de sacarose como tensoativo micelizado foi possível aumentar significativamente não somente a solubilidade aparente da $\mathrm{AmB}$, mas, principalmente sua seletividade para a formação de complexos com o ergosterol, o que poderia diminuir a toxicidade do antibiótico. $\mathrm{O}$ aumento de seletividade foi de cerca de 1000 vezes do que quando a $\mathrm{AmB}$ foi dissolvida em micelas de desoxicolato de sódio (AmB-DOC). Experimentos adicionais com camundongos não infectados, demonstraram que a administração da $\mathrm{AmB}$ em micelas de laurato de sacarose, diminuiu a toxicidade quando comparada com a AmB-DOC, em razão de sua maior seletividade para as células de fungos, preservando sadias as células de mamíferos (9).

Outra estratégia para aumentar a solubilidade da AmB é conjugá-la ao polissacarídeo arabinogalactana (AmB-AG). A alta solubilidade em água, biocompatibilidade, biodegradabilidade e fácil conjugação ao fármaco torna-o um potencial carreador da AmB. Diferentemente das formulações lipídicas, o complexo AmB-AG pode ser facilmente esterilizado por filtração, simplificando o processo de produção (33). 


\section{Associação de AmB-DOC com emulsão de nutri-} ção parenteral (Intralipid $\left.{ }^{\circledR}\right)$. A absorção da $A m B$ por via oral é mínima (inferior a 5\%), e por isso as infecções fúngicas sistêmicas são tratadas através de infusão contínua por via i.v., sendo necessários cuidados especiais com o paciente, como por exemplo, administrar a AmB somente em ambiente hospitalar, por haver necessidade de usar a via i.v. para administração do fármaco (34).

Desde 1956 a AmB é comercializada na forma de pó liofilizado associado ao desoxicolato $\left(\right.$ Fungizon $\left.^{\circledR}\right)$. É reconstituído em soro glicosado formando uma dispersão micelar. A administração i.v. deve ser realizada lentamente para prevenir reações adversas, e caso seja uma administração i.v. Bolus, pode levar a óbito em razão da alta toxicidade do fármaco. Até a metade da década de 90, não havia opção de outras formulações além da AmB-DOC para o tratamento de infecções fúngicas sistêmicas (35).

Na década de 90, em virtude da falta de disponibilidade de formulações lipídicas, a administração da AmB-DOC era veiculada na emulsão de nutrição parenteral Intralipid ${ }^{\circledR}$. Por falta de estudos de compatibilidade e estabilidade, não era conhecido o comportamento dessa formulação. Dessa forma, sua preparação se dava no momento da infusão. A administração desse produto apresentou menos efeitos adversos e uma toxicidade semelhante à formulação convencional (36). Para evidenciar a superioridade da emulsão lipídica de AmB-DOC frente à formulação convencional, novos estudos mostraram que com a utilização da emulsão de AmB-DOC, conseguiu-se a redução dos efeitos tóxicos in vitro por incubação com eritrócitos e in vivo por administração em ratos (37). Uma redução significativa da toxicidade renal da AmB-DOC emulsionada foi obtida em comparação com a formulação convencional AmB-DOC (38).

A partir da década de 90 surgiram as formulações lipídicas, as quais embora tenham demonstrado menor atividade terapêutica, permitiram a administração de doses mais elevadas, com menor toxicidade e melhor perfil nos resultados do tratamento (17).

Anfotericina B Lipossomal (Ambisome ${ }^{\circledR}$ ). Lipossomas convencionais são utilizados para transportar, aumentar o índice terapêutico, podendo também aumentar a solubilidade e proteger os fármacos contra a degradação (Figura 3). Contendo uma ou mais estruturas de bicamadas lipídicas separadas por fases aquosas e uma fase aquosa central, podem encapsular substâncias lipofílicas na bicamada lipídica e substâncias hidrofílicas nas fases aquosas $(39,40)$ (Figura 4).
A primeira formulação lipossomal de $\mathrm{AmB}$ foi proposta no ano de 1984 (41). O AmBisome ${ }^{\circledR}$ é uma preparação de AmB em lipossomas (Lip-AmB) uma ou duas bicamadas lipídicas, pequenas e esféricas, estrututradas a partir de $213 \mathrm{mg}$ de fosfatidilcolina hidrogenada de soja (PCSH), 52mg de colesterol (CHO), $84 \mathrm{mg}$ de dimiristoilfosfatidiglicerol (DMPG), 900mg de sacarose, $0,64 \mathrm{mg}$ de tocoferol como antioxidante e $27 \mathrm{mg}$ de succinato de sódio hexahidratado como constituinte do sistema tampão (42).

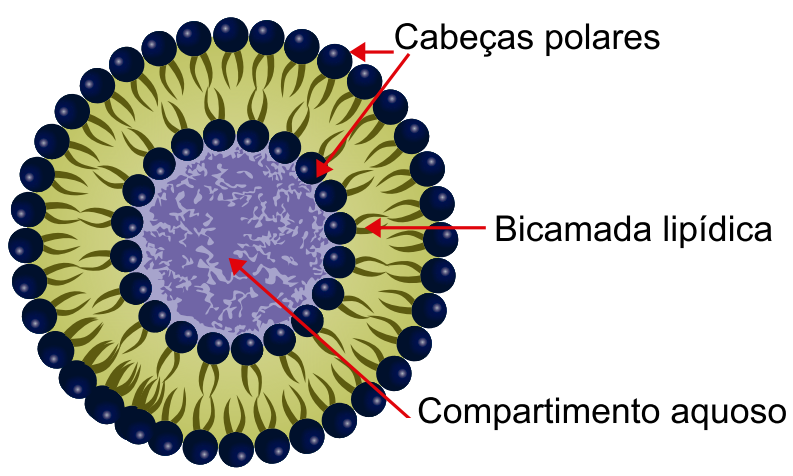

Figura 3 - Representação esquemática de um lipossoma unilamelar. Adaptado de Horiba, 2015 (41).

Foi aprovada pelo FDA em 1997, sendo comercializada na forma de pó liofilizado, o qual deve ser diluído em água para injeção. Embora os lipossomas sejam rapidamente removidos da corrente circulatória, o Lip-AmB proporciona uma biodistribuição diferenciada da $\mathrm{AmB}$, permitindo a redução significativa da toxicidade (35).

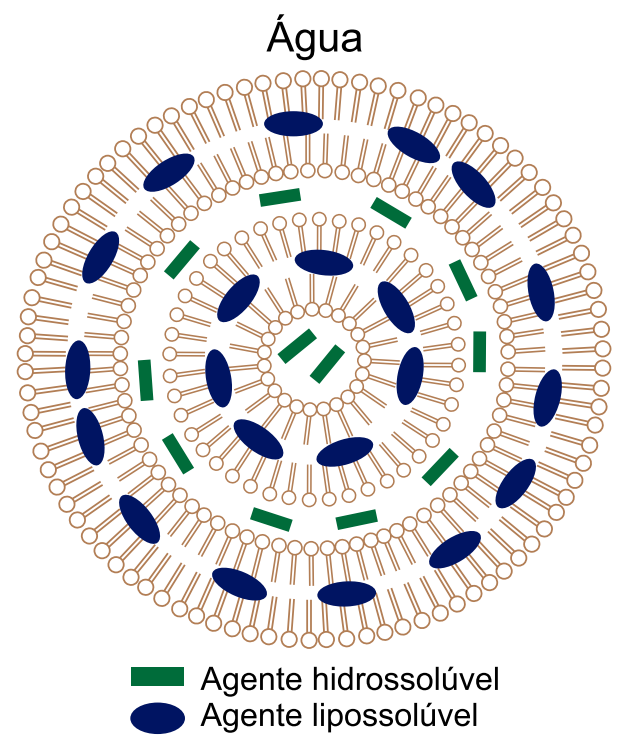

Figura 4 - Estrutura esquemática da AmB lipossomal, AmBisome ${ }^{\circledR}$, demonstrando os possíveis sítios de solubilização para substâncias hidro ou lipossolúveis como a AmB. Adaptado de Hiemenz \& Walsh, 1996 (43). 
Em 1999, Barquist e cols (44) publicaram um ensaio clínico constatando que a Lip-AmB está associada com menor nefrotoxicidade do que a AmB-DOC. Em 2000, Wingard e cols (45) publicaram um estudo duplo cego randomizado com 244 pacientes divididos em três grupos: AmB-DOC, Lip-AmB e complexos lipídicos de AmB (ABLC). Os grupos tiveram sucesso terapêutico similar, mas o grupo relacionado à AmB-DOC teve maiores efeitos colaterais e maior nefrotoxicidade $(42,3 \%)$, além de um maior número de pacientes ter necessitado interromper o tratamento em razão dos efeitos adversos. Um estudo clínico realizado com pacientes com câncer e infecções fúngicas mostrou que a Lip-AmB foi melhor tolerada do que a AmB-DOC (9). Em 2002, Johnson e cols (46), publicaram que pacientes tratados com Lip-AmB tiveram maior sobrevida comparados àqueles pacientes que receberam AmB-DOC.

Para reforçar a superioridade da Lip-AmB sobre a AmB-DOC, lipossomas têm sido usados como veículos para a AmB em tratamentos de criptococose e candidíase, nos quais mostrou-se tão eficiente quanto a AmB-DOC. Além de ser menos tóxica, podem ser administradas doses maiores, que além de realizar um tratamento mais eficaz, também aumenta a qualidade de vida do paciente, por aumentar o intervalo de administração das doses (9). A principal dificuldade está relacionada com o aumento considerável do custo do tratamento com Lip-AmB. Em média, o custo de um miligrama de AmB na formulação lipossomal custa 35 vezes mais que um miligrama de AmB-DOC, ou seja, apesar dos benefícios o valor do tratamento limita o seu emprego, principalmente para atender regiões de baixa renda.

Anfotericina B em Dispersão Coloidal (ABCD). Em 1996 o FDA aprovou o Amphocil ${ }^{\circledR}$, uma dispersão coloidal de AmB (ABCD), para o tratamento de pacientes refratários ou intolerantes à AmB-DOC. Tecnicamente, trata-se de uma dispersão coloidal de AmB em sulfato sódico de colesterila, na razão molar 1:1, estruturalmente organizada como partículas discóides ou microdiscos com diâmetro médio de 122 nm (47) (Figura 5).

Um estudo clínico duplo cego aleatório comparando $A B C D$ e AmB-DOC foi realizado com 174 pacientes com infecção invasiva de aspergilos. Não houve diferença estatística na resposta terapêutica, mas houve redução significativa da nefrotoxicidade nos pacientes tratados com $\mathrm{ABCD}$. A proporção foi de cerca de $25 \%$ de nefrotoxicidade nos pacientes tratados com $A B C D$, contra $49 \%$ de nefrotoxicidade para os pacientes que foram tratados com a AmB-DOC (49).

\section{Complexo Lipídico de Anfotericina B (ABLC).} No ano de 1995, surgiu o Abelcet ${ }^{\circledR}$, um complexo lipídico constituído de diesteroilfosfatidilcolina (DMPC) e diesteroilfosfatidilglicerol (DMPG), na razão molar 7:3 e contendo $36 \mathrm{~mol} \%$ de AmB. É um sistema multilamelar do tipo "ribbon-linke", formado por partículas em formato de roseta de fitas (Figura 6), tendo sido esta a primeira formulação lipídica contendo AmB aprovada pelo FDA (17).

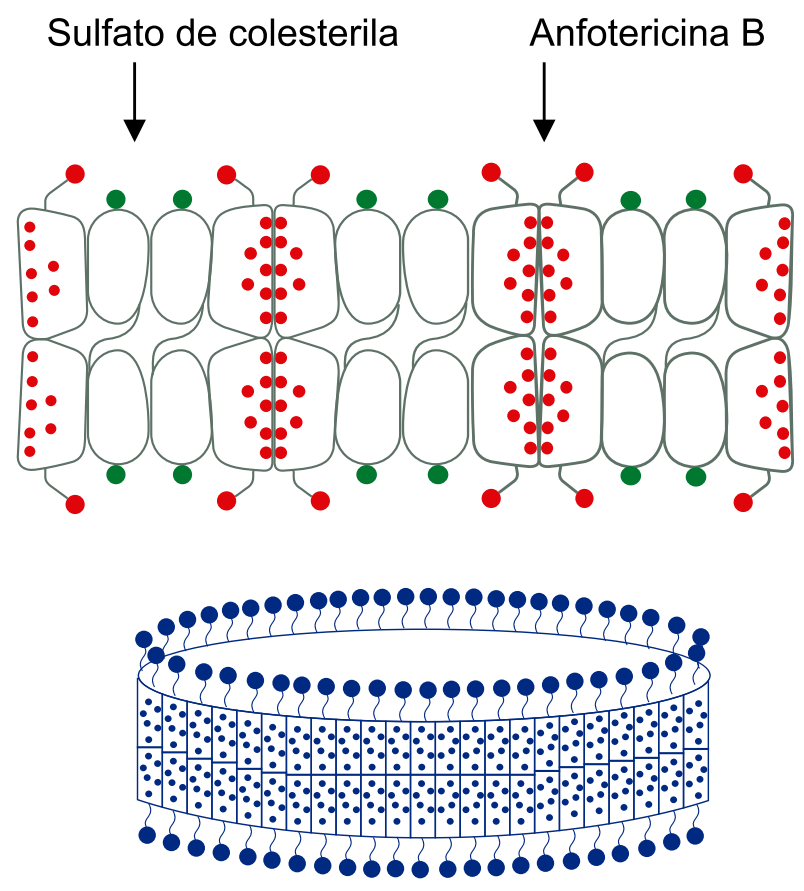

Figura 5 - Modelo da Dispersão Coloidal de AmB do tipo ABCD "Amphotericin B Colloidal Dispersion". Adaptado de Janoff et al., 1993 (48).

Formulações lipídicas de AmB foram desenvolvidas a cerca de quinze anos, apresentando menor toxicidade em relação à medicação convencional. Quando complexada com lipídeos, a AmB atinge maiores concentrações no fígado e no baço do que a AmB-DOC, mas nos rins seus níveis são menores. Isto explica a menor nefrotoxicidade das preparações lipídicas, que são por outro lado, discretamente mais hepatotóxicas do que a medicação convencional. Mesmo pacientes com lesão renal ou com anemia induzida por AmB-DOC, podem completar o tratamento com as formulações lipídicas, em geral sem agravamento destes efeitos adversos. Assim, o principal benefício destas preparações é a maior segurança quanto à toxicidade celular no uso prolongado da $\mathrm{AmB}$ (17) um estudo retrospectivo analisando mais de 500 pacientes idosos imuno comprometidos devido ao tratamento quimioterápico foi realizado comprovando essa hipótese. 


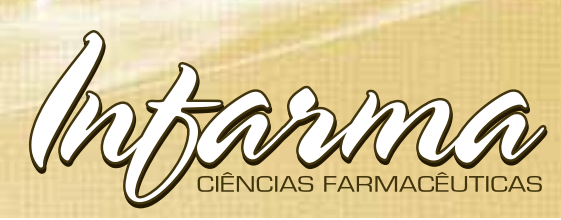

A efetividade e segurança de ABLC foram demonstradas num tratamento de infecção fúngica invasiva. Uma pequena incidência de toxicidade renal foi observada no grupo tratado com ABLC, se comparado com pacientes tratados com formulação convencional AmB-DOC, cuja concentração de creatinina sérica chegou a duplicar antes do final do tratamento. Os autores puderam demonstrar que ABLC e AmB-DOC foram igualmente efetivos, mas que a formulação lipídica pareceu preservar a função renal (50). Mais uma vez a relação custo-benefício das novas formulações de $\mathrm{AmB}$ ainda não oferece vantagens diferenciadas em relação às outras formulações com agregação de tecnologia, que possam estimular à adesão ao tratamento com esse tipo de formulação, principalmente se tratando do atendimento pelo sistema publico de saúde (33).

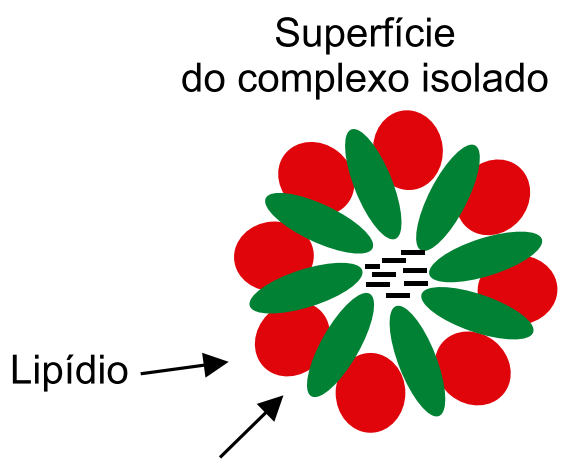

Anfotericina B

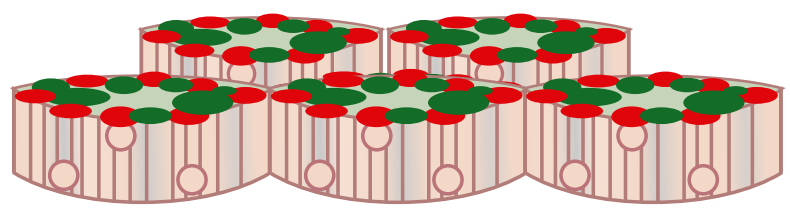

\section{Complexos associados}

Figura 6 - Modelo do complexo lipídico ABLC "Amphotericin B Lipid Complex". Adaptado de Hiemenz \& Walsh, 1996 (43).

\section{TOXICIDADE DA AMB}

Desde 1956, no início do uso clínico da AmB, já havia na literatura médica descrições sobre os efeitos tóxicos para os rins devido ao uso desse antibiótico. $\mathrm{O}$ primeiro estudo específico que abordou a toxicidade renal da AmB indicava a redução da filtração glomerular, hipocalemia e hipomagnesemia (51).

Embora o mecanismo de nefrotoxicidade ainda não esteja completamente elucidado, a literatura mostra que a toxicidade pode estar ligada ao estado físico da
AmB. A nefrotoxicidade também é apontada como consequência de uma série de mecanismos incluindo lesões em razão da vasoconstrição das arteríolas renais e consequente redução do aporte de sangue aos túbulos renais e aos glomérulos, bem como a uma ação lítica das lisozimas que atacam membranas ricas em colesterol das células dos tubos renais (52). Também são descritos vários fatores que podem induzir a nefrotoxicidade, incluindo dose administrada, duração da terapia, nível de creatinina no plasma e o uso concomitante com outros fármacos potencialmente nefrotóxicos. Pacientes transplantados e imunocomprometidos em geral estão entre os mais vulneráveis a nefrotoxicidade causada pela $\mathrm{AmB}$ (53).

Estudos de modificações moleculares da AmB tem sido realizados com o intuito de diminuir a toxicidade e aumentar a sua solubilidade. Na década de 70 foi introduzido o metil-éster da $\mathrm{AmB}$, solúvel em água, além de diminuir a nefrotoxicidade. Porém, o uso foi limitado pelo surgimento de leucoencefalopatia (54).

A veiculação de $\mathrm{AmB}$ em sistemas do tipo lipossoma (item 2.1.2.) foi uma estratégia que buscava tanto a melhoria da solubilidade do fármaco, mas também apresentou vantagens relacionadas à redução de toxicidade, principalmente atribuídas à modificação da superfície externa dos lipossomas com cadeias de polietilenoglicol (PEG), ou peguilação (Figura 7).

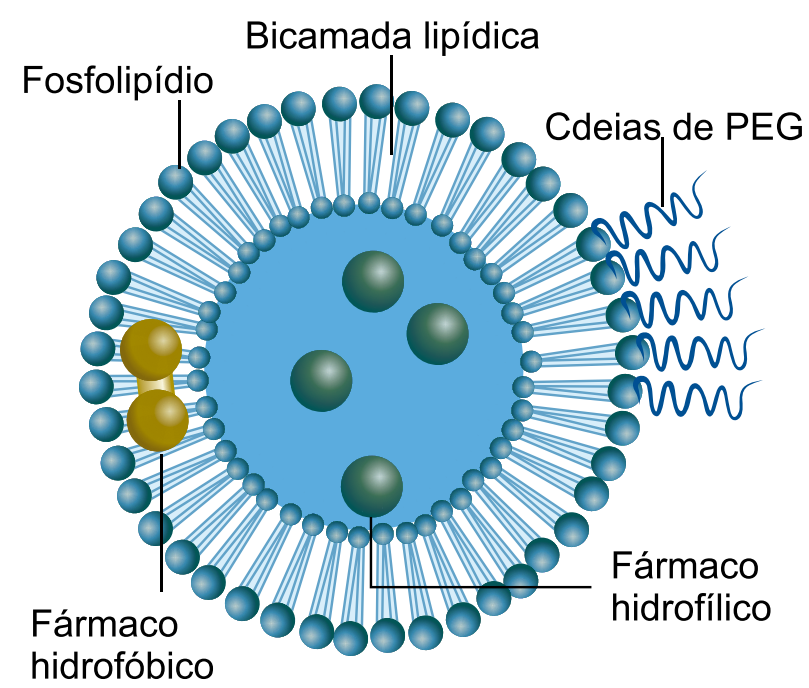

Figura 7 - Encapsulação de fármacos hidrofílicos e lipofíflicos em lipossoma unilamelar com superfície modificada por peguilação. Adaptado de Deshpande et al., 2013 (56)

A peguilação foi introduzida como meio de prolongar o tempo de meia-vida do fármaco na corrente circulatória e reduzir a toxicidade em formulações que usam lipossomas como estratégia de administração. Isto 
pode ser explicado pelo fato de que as cadeias polietilênicas projetam-se para o exterior da estrutura, criando uma região hidrodinâmica na superfície dos lipossomas, causando um impedimento estérico nessa superfície e protegendo a bicamada lipídica da opsonização e remoção da corrente circulatória (55). Essa estratégia levou a uma substancial redução da toxicidade, comparada com o efeito do lipossoma convencional e a um aumento da eficácia terapêutica em razão do maior tempo de residência dos lipossomas peguilados na corrente circulatória $(13,55)$.

Os avanços obtidos por grupos de pesquisas nos últimos anos apontam apara perspectivas favoráveis no tratamento de infecções fúngicas sistêmicas. As tecnologias existentes transcendem as disponíveis no mercado, apresentadas neste trabalho e seguem varias tendências distintas, desde as vias de administração até o tipo dos sistemas de administração estudados. Sistemas como microemulsões e nanopartículas e complexos moleculares com as diferentes ciclodextrinas estão entre os mais utilizados, em alguns caos com avanços bastante significativos, podendo se tornar alternativas reais na terapia antifúngica no futuro.

\section{CONCLUSÃO}

Apesar da elevada toxicidade da AmB e da introdução de antifúngicos azólicos sistêmicos na década de 1980, a potência, o espectro de ação e os quase 50 anos de experiência clínica têm assegurado que a AmB permaneça como fármaco de escolha no tratamento das infecções fúngicas sistêmicas que acometem principalmente pacientes imuno comprometidos. Em razão da alta toxicidade da AMB-DOC, as formulações comerciais da anfotericina $\mathrm{B}$, estruturadas com lipídios foram introduzidas no mercado farmacêutico estando em uso clínico. Embora essas formulações lipídicas tenham demonstrado pouco menor atividade antifúngica, a diminuição da toxicidade permitiu a administração de doses mais elevadas, obtendo-se melhores resultados clínicos no tratamento.

A melhor eficiência desses sistemas de administração proporciona menor período de resposta terapêutica, além de exibir menor toxicidade em comparação com a formulação convencional de Fungizon ${ }^{\circledR}$. Os estudos com as formulações lipídicas, como os complexos lipídicos e lipossomas, mostraram eficácia e segurança similares tanto para pacientes imunocomprometidos, como no tratamento profilático em pacientes imunologicamente normais. Apesar da comprovada superioridade de tais formulações, o alto custo tem restringido o seu uso, principalmente em regiões endêmicas pobres. No entanto, entre as opções de formulações mais eficazes, ainda é necessário avaliar e decidir se é conveniente ena expor os pacientes à toxicidade significativa da AMB-DOC, em troca da diminuição dos custos da terapia com esse medicamento.

\section{REFERÊNCIAS}

1. Lacaz CS, Sampaio SAP. Tratamento da Blastomicose Sul- Americana com Anfotericina B. Resultados Preliminares. São Paulo Med J. 1958; 52: 443-50.

2. Gold N, Stout, HA, Pagano JF.; Donovick R. Amphotericin A and B, antifungal antibiotics produced by a streptomycete. I. In vivo studies. Antibiotics annual. 1956; 1 : 579-86.

3. Dutcher JD, Gold W, Pagano JF, Vandeputte, J. Amphotericin B, its production, and its salts US patent 2908611 A, 1959, Oct. 13.

4. Esposito E, Bortolotti F, Menegatti E, Cortesi R. Amphiphilic association systems for Amphotericin B delivery. Int J Pharm. 2003; 260: 249-60. DOI: 10.1016/S03785173(03)00270-9.

5. Hereć M, Dziubińska H, Trebacz K, Morzycki JW, Gruszecki WI. An effect of amphotericin B on transport across model lipd membranes and tonoplast membranes. Biochem Pharmacol. 2005; 1;70(5):668-75 DOI: 10.1016/j.bcp.2005.06.001.

6. Amato VS, Tuon FF, Bacha HA, Amato Neto V, Nicodemo A Mucosal leishmaniasis. Current scenario and prospects for treatment. Acta Tropica. 2008; 105: 1-9. DOI: 10.1016/j.actatropica.2007.08.003.

7. Wu TC. On the development of antifungal agents: perspective of the U.S. Food and Drug Administration. Clin Infect Dis. 1994; 19: 54-8. doi:10.1093/clinids/19.Supplement_1.S5.

8. Dismukes WE. Introduction to antifungal drugs. Clin Infect Dis. 2000; 30(4):653-7. DOI: 10.1086/313748.

9. Brajtburg J, Powderly WG, Kobayashi GS, Medoff G. Amphothericin B: Delivery Systems. Antimicrob Agents Ch. 1990; 34(3): 381-4. DOI: 0066-4804/90/03038104\$02.00/0.

10. Bolard J, Joly V, Yeni P. Mechanism of action of amphotericin B at the cellular level. It's odulation by delivery system. J Liposome Res. 1993; 3: 409-27. DOI: $10.3109 / 08982109150728$. 
11. Laniado-Laborín R, Cabrales-Vargas MN. Amphotericin B: side effects and toxicity. Rev Iberoam Micol. 2009; 31;26(4):223-7. DOI: 10.1016/j.riam.2009.06.003.

12. Whyte BS, Peterson RP, Hartsel SC. Amphotericin B and nystatin show different activities on sterol free vesicles. Biochem Biophys Res Commun. 1989. 164: 60914. DOI: 10.1016/0006-291X(89)91503-9.

13. Moribe K, Maruyama K. Pharmaceutical design of the liposomal antimicrobial agents for infectious disease. Curr Pharm Des. 2002; 8(6):441-54. DOI: $10.2174 / 1381612023395853$

14. Asher IM, Schurartzman G. Amphotericin B. Anal Profiles Drug Subst. 1977. 6:1-42.

15. Araújo IB. Novos Sistemas Carreadores para Anfotericina B: Estudo dos Parâmetros Tecnológicos e Fármaco-tóxicológicos. [Tese] - Natal, Centro de Ciências da Saúde, UFRN, 2005.

16. Larabi M, Gulik A, Dedieu JP, Legrand P, Barratt G, Cheron $M$. New lipid formulation of amphotericin B: spectral and microscopic analysis. BBA-Rev Biomembranes. 2004; 1664: 172-81. DOI: 10.1016/j.bbamem.2004.05.003.

17. Filippin FB, Souza LC. Eficiência terapêutica das formulações lipídicas de anfotericina B. Rev Bras Ciênc Farm. 2006; 42:167-94. DOI: 10.1590/S151693322006000200003.

18. Golenser J, Domb A. New Formulations and Derivatives of Amphotericin B for Treatment of Leishmaniasis. Mini-Rev Medic Chem. 2006; 153-62. DOI: 10.2174/138955706775476037.

19. Oliveira AG, Chiavacci LA, Scarpa MV, Egito EST. Microemulsions: physico-chemical approaches on the system for pharmaceutical applications. In: Li SJ, editor. Current Focus on Colloids and Surfaces. Kerala, India: Transworld Research Network; 2009; 1: 57-84.

20. Oliveira AG, Scarpa MV, Correa MA, Cera LFR, Formariz, TP. Microemulsões: estrutura e aplicações como sistema de liberação de fármacos. Quim. Nova. 2004; 27 : 131-138. DOI: 10.1590/S0100-40422004000100023

21. Formariz TP, Chiavacci LA, Sarmento VHV, Franzini CM, Silva-Jr AA, Scarpa MV, Santilli CV, Egito EST, Oliveira AG. Structural changes of biocompatible neutral microemulsions stabilized by mixed surfactant containing soya phosphatidilcholine and their relationhship with doxorubicin release. Colloids Surf B Biointerfaces. 2008; 63:287-95. DOI: 10.1016/j.colsurfb.2007.12.021.

22. Silva GBRF, Scarpa MV, Rossanezi G, Egito EST, Oliveira AG. Development and characterization of biocompatible isotropic and anisotropic oil-in-water colloidal dispersions as a new delivery system for methyl dihydrojasmonate antitumor drug. Int J Nanomed. 2014; 9:867-76. DOI: 10.2147/IJN.S46055.

23. Franzini MC, Pestana KC, Molina EF, Scarpa MV, Egito EST, Oliveira AG. Structural properties induced by the composition of biocompatible phospholipid-based microemulsion and amphotericin B association. J Biomed
Nanotech. 2012; 8:350-359. DOI: http://dx.DOI. org/10.1166/jbn.2012.1435.

24. Dalmora ME, Dalmora SL, Oliveira AG. Inclusion complexo of piroxicam with beta-cuclodextrin and incorporation in cationic microemulsion. In vitro drug release and in vivo anti-inflammatory effect. Int J Pharm. 2001; 222(1): 45-55. DOI: 10.1016/S0378-5173(01)00692-5.

25. Gutiérrez LO, Fernández RE, Dea-Ayuela MA, Torrado JJ, Fernandez FB, Alunda JM. In vitro effect of new formulations of amphotericin $\mathrm{B}$ on amastigote and promastigote forms of Leishmania infantum.. Int J Ant. Ag. 2007; 30:325-329. DOI: 10.1016/j.ijantimicag.2007.05.013.

26. Baginski M, Resat H, MCCammon JA. Molecular Properties of Amphotericin B Membrane Channel: A Molecular Dynamics Simulation. Mol Pharmacol. 1997; 52: 560-570. DOI: 10.1124/mol.52.4.560.

27. Hartsel SC, Baas B, Bauer E, Foree LT, Kindt-Jr K, Preis H, Scott A, Kwong EH, Ramaswamy M, Wasan KM. Heat induced superaggregation of amphotericin B modifies its interaction with serum proteins and lipoproteins and stimulation of TNF. J Pharm Sci. 2001; 90: 124-33. DOI: 10.1002/1520-6017(200102)90:23.3.CO;2-O.

28. Moreno MA, Frutos P, Ballesteros MP. Lyophilized lecitn-based oilwater microemulsion as a new low toxic delivery sistem for Amphotericin B. Pharm Res. 2001; 18: 344-51. DOI: 0724-8741/01/0300-0344\$19.50/0.

29. Fukui H, Koike T, Saheki A, Sonoke S, Seki J. A novel delivery system for amphotericin B with lipid nano-sphere (LNS $\left.{ }^{\circledR}\right)$. Int J Pharm. 2003; 265:37-45. DOI:10.1016/S0378-5173(03)00404-6.

30. Shao PL, Huanga LM, Hsueh PR. Recent advances and challenges in the treatment of invasive fungal infections. Int J Antimicrob. Agents. 2007. 30:487-95. DOI: 10.1016/j.ijantimicag.2007.07.019.

31. Adediran SA, Day TP, Sil D, Kimbrell MR, Mamali S, David SA. Synthesis of a Highly Water-Soluble Derivative of Amphotericin B with Attenuated Proinflammatory Activity. Mol Pharmaceutics. 2009; 6(5): 1582-90. DOI: $10.1021 / \mathrm{mp} 9001602$.

32. Torrado-Durán JJ, Torrado-Durán S, Sánchez-Brunete JA, Bolás- Fernández F, Dea-Ayuela MA, Rama-Iñiguez $\mathrm{S}$, et al. Microesferas de anfotericina B. Solicitud de Patente, Oficina Espanõla de Patentes y Marcas, 2004.

33. Golenser J, Frakenburg S, Ehenfreund T, Domb JA. Efficacious Treatment of Experimental Leishmaniasis with Amphotericin B-Arabinogalactan Water-Soluble Derivatives. Antimicrob Agents Ch. 1999; 43(9):2209-2214. DOI: 0066-4804/99/\$04.00+0.

34. Bennett JE. Fármacos antimicrobianos. In: Goodman \& Gilman. As Bases Farmacológicas da Terapêutica. 10 ed. Rio de Janeiro: McGraw Interamericaca do Brasil Ltda, 2003. cap. 49, p.971-974.

35. Kleinberg $\mathrm{M}$. What is the current and future status of conventional amphotericin B? Int J Antimicrob Agents. 2006; 27:12-6. DOI: 10.1016/j.ijantimicag.2006.03.013. 
36. Galera RML. Anfotericina B: determinación em diversos fluidos biológicos por cromatografia líquida. Aplicación a estudios famacocineticos y de estabilidad química.[Tese]. Barcelona - Unidad de Monitorización de Fármacos del Servicio de Bioquímica del Hospital General Vall d'Hebron de Barcelona.2000.

37. Souza LC, Campa A. Pharmacological parameters of intravenously administered amphotericin B in rats: comparison of the conventional formulation with amphotericin B associated with a triglyceride-rich emulsion. J Antimicrob Chemother. 1999; 44: 77-84. DOI: 10.1093/ jac/44.1.77.

38. Souza LC, Saldiva PHN, Campa A. Lipid emulsion reduces subacute tocixity of amphotericin B: a histopathological study. Exp Toxic Pathol. 2000; 52:169-175. DOI: 10.1016/S0940-2993(00)80115-3.

39. Lopez-Berestein G, Hopper RL, Mehta R, Mehta K, Hersh EM, Juliano, RL. Liposome-encapsulated amphotericin B for the treatment of disseminated candidiases in neutropenic mice. J Infect Dis. 1984; 150:278-283. DOI: 10.1093/infdis/150.2.278.

40. Storm G, Etten EV. Biopharmaceutical aspects of lipid formulations of amphotericin B. J. Clinic Microbiol Infect Dis. 1997; 16: 64-73. DOI: 10.1007/BF01575123.

41. Horiba Scientific, Particle Size Analysis of Liposomes using Dynamic Light Scattering. In:http://www. horiba.com/in/scientific/products/particle-characterization/applications/ pharmaceuticals/ liposomes, acessado em 15 outubro 2015.

42. BRASIL, Agência Nacional de Vigilância Sanitária ANVISA, AmBisome ${ }^{\circledR}$ Injetável, Informações Técnicas aos Profissionais da Saúde, http://www.anvisa.gov.br/ datavisa/fila bula/frmVisualizarBula.asp? pNuTransa$\mathrm{cao}=11075782014 \&$ pIdAnexo $=2356577$.

43. Hiemenz JW, Walsh TJ. Lipid Formulations of Amphotericin B: Recent Progress and Future Directions. Clin Infect Dis. 1996; 22(Suppl):S133-44. DOI: 10.1093/clinids/22.Supplement_2.S13.

44. Barquist E, Fein E, Shadick D, Johnson J, Clark J, Shatz D. A randomized prospective Trial of amphotericin B lipid emulsion versus dextrose colloidal solution in critically patients. J Trauma. 1999; 47(2):336-40. DOI: 10.1097/00005373-199908000-00020.

45. Wingard JR, White MH, Anaissie E, Raffalli J, Goodman J, Arrieta A. A randomized, double-blind comparative trial evaluating the safety of liposomal amphotericin $B$ versus amphotericin B lipid complex in the empirical treatment of febrile neutropenia. Clin Infec. Dis. 2000; 31:1115-1163.DOI: 10.1086/317451.
46. Johnson PC, Wheat LJ, Cloud GA, Goldman M, Lancaster D, Bamberger DM. Safety and efficacy of liposomal amphotericin B compared with conventional amphotericin for induction therapy of histoplasmosis in patients with AIDS. Ann Intern Med 2002; 137:105-109. DOI: 10.7326/0003-4819-137-2-200207160-00008.

47. Robinson RF, Nahata MC. A comparative review of conventional and lipid formulations of amphotericin B. J Clin Pharm Ther. 1999; 24(4):249-257. DOI: 10.1046/j.1365-2710.1999.00220.x.

48. Janoff AS, Perkins WR, Saleton SL, Swenson CE. Amphotericin B lipid complex (ABLC ${ }^{\mathrm{TM}}$ ): a molecular rationale for the attenuation of amphotericin Brelated toxicities. J Lip Res. 1993; 3: 451-472. DOI: 10.3109/08982109309150730.

49. Bowden R, Chandrasekar P, White MH, Li X, Pietrelli L, Gurwith M, et al. A Double-Blind, Randomized, Controlled Trial of Amphotericin B Colloidal Dispersion versus Amphotericin B for Treatment of Invasive Aspergillosis in Immunocompromised Patients. Clin Infect Dis. 2002; 35(4):359-366. DOI: 10.1086/341401.

50. Hooshmand-Rad R, Chu A, Gotz V, Morris J, Batty $\mathrm{S}$, Freifeld A. Use of amphotericin B lipid complex in elderly patients. J Infection. 2005; 50: 277-287. DOI: 10.1016/j.jinf.2004.07.004.

51. Butler WT, Bennett JE, Alling DW, Wertlake PT, Utz J, Phill GJ. Nephrotocicity of amphotericin B, early and late events in 81 patients. Ann Intern Med. 1964; 61:175-187. DOI: 10.7326/0003-4819-61-2-175.

52. Yoo BK, Jalil MA, Lee ES, Han K. Reduced Renal Toxicity of Nanoparticular Amphotericin B Micelles. Prepared with Partially Benzylated Poly-L-aspartic acid. Biol Pharm Bull. 2006; 29(8): 1700-1705. DOI: 10.1248/bpb.29.1700.

53. Alexander BD, Wingard JR. Study of Renal Safety in Amphotericin B Lipid Complex-Treated Patients. Clin Infect Dis. 2005; 40:414-21. DOI: 10.1086/429335.

54. Berdichevski RH. Nefrotoxicidade da AmB associada a pacientes de baixo risco. [Dissertação] Porto Alegre. Faculdade de Medicina. UFRGS,2003.

55. Jadhav MP, Nagarsenker MS, Gaikqard RV, Samad A, Kshirsagar NA. Formulation and evaluation of long circulating liposomal amphoterincin B: A scinti-kinect study Tc in BALB/C mice. Indian J Pharm Sci. 2011; 73(1): 57-64. DOI: 10.4103/0250-474X.89757.

56. Deshpande PP, Biswas S, Torchilin VP. Current Trends in the Use of Liposomes for Tumor Targeting. Nanomedicine. 2013; 8(9):1509-1528. DOI: 10.2217/nnm.13.118. 